\title{
MULTI-SCALE SPACE VEHICLE COMPONENT IDENTIFICATION
}

\author{
William W. L. Lam, Clement C. C. Pang and Nelson H. C. Yung \\ Department of Electrical and Electronic Engineering \\ The University of Hong Kong \\ Pokfulam, Hong Kong SAR, China
}

\begin{abstract}
Vision bascd velicle recognition systems have an important role in traffic surveillance. Most of these systems however fail to distinguish vehicles with similar dimensions due to the lack of other details. This paper presents a new scale space method for identifying components of moving vehicles to cnable recognition eventually. In the proposed method, vehicles are first divided into multi-scale regions based on the center of gravity of the foreground vehicle mask. It utilizes both the texture scale space and the intensity scale space to determine regions that are homogenous in texture and intensity, from which velucle components are identified based on the relations between these regions. This method was tested on over a hundred outdoor traffic images and the results are very promising.
\end{abstract}

\section{INTRODUCTION}

In visual traffic surveillance, most vehicle recognition systems simply distinguish the size, dimension, shape or contour of the vehicle, from which vehicles can be categorized by type $[3,4,6,7]$. Gupte et al. [3] classified vehicles based on a non-rigid vehicle model, while Lai et al. [4] classified vehicles based on dimension. Tan et al. [6] extracted vehicle by matching model discrimination, and Wei et al. [7] classified vehicles using a parameterized model and neural networks. With improvement from [4], Fung et al. [1] additionally considered vehicle shape for classification based on vehicle motion.

Broadly, these techniques addressed the problem of classifying vehicles of the same size or dimension. However, if different vehicles of similar dimension are present, these methods become inadequate, as similar sized vehicles will most likely be recognized as one class because of the non-utilization of vehicle details. Recently high-tech surveillance cameras are emerging and providing high resolution images. This motivates us to

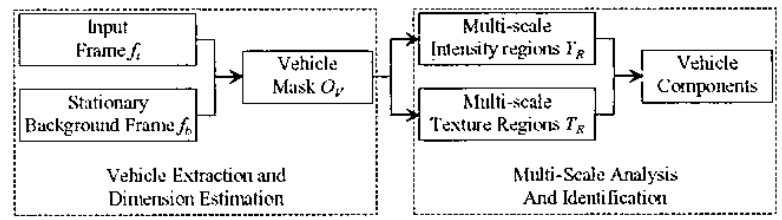

Fig. I: Scale space identification of vehicle components

consider decomposing the velicle object into components such as roof, bonnet, wheels and etc, as an alternative way to discriminate between different velicles. In this paper, we propose a scale space method as depicted in Fig. 1 for this purpose. Assuming that the vehicle is in motion, we first employ the extraction method as described in [5]. From each cxtracted vehicle, we then estimate its dimension to identify similar sized vehicles and compute its texture scale space (TSS) and intensity scale space (ISS) based on the centers of gravity. By fusing the texture and intensity regions corresponding to a selected scale, vehicle components can be subsequently identified. Details of the proposed method are presented in Section 2, and simulation results and discussion are given in Section 3, where conclusions are drawn in Section 4.

\section{PROPOSED METHOD}

It is further assumed that the surveillance camera is roadside mounted and stationary, while the light source can be single and strong (as in day time) or multiple and diffused (as in night time). Based on these assumptions, the proposed method comprises of: (1) vehicle extraction and dimension estimation; (2) multi-scale analysis and identification.

\subsection{Vehicle Extraction and Dimension Estimation}

In vehicle extraction, we first extract the moving vehicles from the stationary background using a texture-based method described in [5]. This method considers the textural difference between the vehicle and other road features and its merits are that it is accurate in generating a vehicle mask (outline describing the vehicle) and able to 
eliminate shadow and reflection on the velicle chassis. Then, veluicle dimensions are estimated using the deformablc model approach as described in [4], which relies on a set of calibrated camera parameters [2]. This sct of calibrated camera parameters is useful in providing the transfonmation between the 3D world coordinates and the 2D image coordinates as seen by the camera. Vehicle dimensions including width, length and height are subsequently calculated in 3D coordinates. This estimation method enables us to identify vehicles of similar dimensions for identification in the next step.

\subsection{Multi-Scale Analysis and Identification}

After the vehicles have been extracted, the vehicle mask $O_{F}$ of cach similar sized veluicle is constructed by segmenting the boundary of the vchicle through the subtraction between the input frame $f_{i}$ and the stationary background frame $f_{b}$. $O_{F}$ is symbolized in Fig. 2(a) by the whitc ellipse. Fig. 2(b-d), illustrate the concept of scale space by partitioning $O_{V}$. into quarters each times (as in quad-tree methods), using the center-of-gravity (CG) algorithm. This algorithm computes the $\mathrm{CG}$ of each region (or sub-region) and divides the region horizontally and vertically into four parts centered at the CG. If $O_{V}$ is defined as root region $R_{0}$ as shown in Fig. 2(a), then this root region divides into four scale 1 sub-regions $R_{0,0} . R_{0,1}$, $R_{0.2}, R_{0,3}$ as shown in Fig. 2(b) and their corresponding pixels within the vehicle mask $O_{V}$ are determined as,

$$
\begin{gathered}
\sum_{0, \ldots, n} i \cdot O_{V}(i, j) \quad \frac{\sum_{i, j \in R_{0, \ldots}} j \cdot O_{V}(i, j)}{\sum_{i, j \in R_{0, \ldots, n}} O_{V}(i, j)} \quad y_{0, \ldots, n}=\frac{\sum_{i, j \in R_{0, \ldots, n}}}{\sum_{i, j \in R_{0, \ldots, n}} O_{V}(i, j)} \\
x_{0, \ldots, n, 0}(i, j) \forall i \leq x_{0, \ldots, n}, j \leq y_{0, \ldots, n} \text { and } i, j \in R_{0, \ldots, n} \\
R_{0, \ldots, n, 1}(i, j) \forall i \leq x_{0, \ldots, n}, j>y_{0, \ldots, n} \text { and } i, j \in R_{0, \ldots, n} \\
R_{0, \ldots, n, 2}(i, j) \forall i>x_{0, \ldots, n}, j \leq y_{0, \ldots, n} \text { and } i, j \in R_{0, \ldots, n} \\
R_{0, \ldots, n, 3}(i, j) \forall i>x_{0, \ldots, n}, j>y_{0, \ldots, n} \text { and } i, j \in R_{0, \ldots, n}
\end{gathered}
$$

where $n$ is the sub-region location, $i, j$ are the position displacements and $x_{0, \ldots, n,}, y_{0, \ldots, n}$ are the central pixel of region $R_{0, \ldots, n}$. The consequent sub-regions $R_{0, \ldots, n}$ can be further partitioned as depicted in Fig. 2(c)-(d). These square like multi-scale regions are useful in comparing visual properties between vehicle components of inconsistent sizes, i.e., the bonnet of the same vehicle that appear to be of different size as seen in different frames within an image sequence.

After partitioning $O_{V}$ into multi-scale regions, the intensity courier $Y_{R}$ of each sub-region $R_{0, \ldots, n}$ is determined based on intensity averaging.

$$
Y_{R_{0_{\ldots, n}, n}}=\frac{\sum_{i, j \in R_{0_{0} \ldots, n}} f_{i}(i, j)}{\sum_{i, j \in R_{0_{n}, \ldots, n}} O_{V}(i, j)}
$$
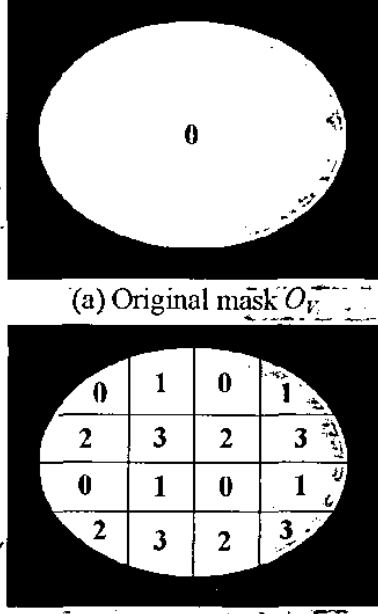

(c) Scale 2 (16 sub-regions) (a) Original mask $O_{V}$

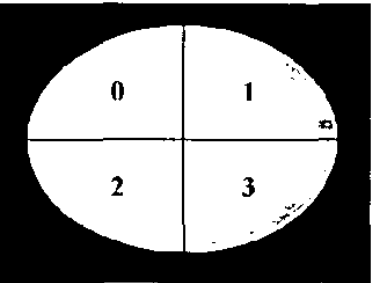

(b) Sçale 1 (4 sub-regions)

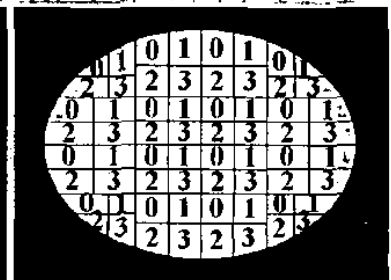

(d) Scäle 3 (64 sub-regions)
Fig. 2: Illustration of the partitioning of a mask by the center-ofgravity algorithm

and the textual courier $T_{R}$ of each sub-region $R_{0} \ldots, n$ is calculated based on intensity variance,

$$
T_{R_{0, \ldots, n}}=\frac{\sum_{i, j \in R_{0, \ldots, n}}\left(f_{i}(i, j)-Y_{R_{0, \ldots, n}}\right)^{2}}{\sum_{i, j \in R_{0, \ldots, n}} O_{V}(i, j)} .
$$

Comparing to traditional edge detector of segmentation, our intensity $Y_{R}$ and textural $T_{R}$ couriers arc helpful in sorting out vehicle components as each vehicle component has comparative intensity or textural relationship with others. From all the $T_{R}$ at each scale, the mean $T_{S}$ and moving average $\bar{T}_{S}$ are calculated by:

$$
T_{S}=\frac{\sum_{n \in S} T_{R_{0, \ldots, n}}}{4^{S}} \overline{T_{S}}=\frac{1}{S+1} \sum_{k=0}^{S} T_{k},
$$

where $S$ is the scale. At texture scale zero, $T_{S}$ is the largest and continues to decrease in subsequence scales until $T_{S}$ becomes zero for the smallest sub-region $R_{0, \ldots, n}$. The mean $Y_{S}$ of all the $Y_{R}$ at each scale is also calculated by:

$$
Y_{S}=\frac{\sum_{n \in S} Y_{R_{0 \ldots . n}}}{4^{S}} .
$$

To find a suitable scale for identification, we define the critical textural scale $C_{S}$, which is chosen when the difference between $\bar{T}_{S}$ and $T_{S}$ is maximum,

$$
S=C_{S} \text { if } \max \left(\overline{T_{S}}-T_{S}\right) .
$$

As depicted in Fig. $3, \max \left(\overline{T_{S}}-T_{S}\right)$ corresponds to a scale ( 6 in this case) that other scales below or above it gives a smaller variance. If scale lower than $C_{S}$ is chosen, the component characteristics are insufficient to warrant an accurate identification. On the other hand, if scale higher than $C_{S}$ is chosen instead, too many superfluous 


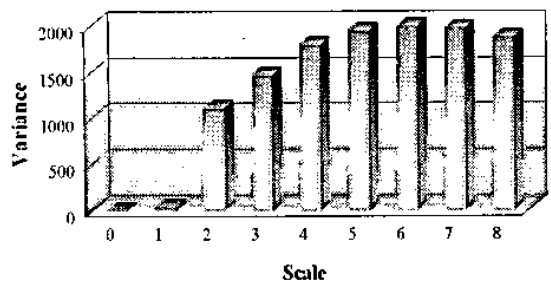

Fig. 3: $\overline{T_{S}}-T_{S}$ on each scale of the sedan shown in Fig. 4(a)

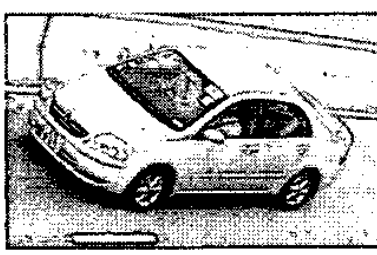

(a) Input frame $f_{i}$

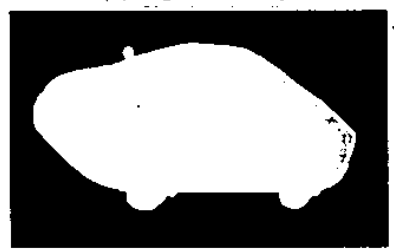

(c) Vehicle mask $O_{V}$

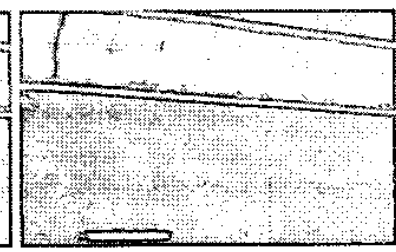

(b) Background frame $f_{b}$

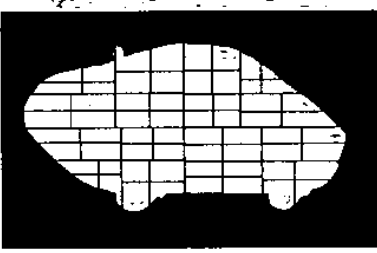

(d) Scale 3 regions
Fig. 4: Partitions on a gray color sedan

features have to be computed and discriminated. Once $C_{S}$ is determined, vehicle components can be segmented by comparing the related intensity $Y_{R}$ and textural $T_{R}$ couriers on the critical textural scale $C_{S}$. Such components include body (roof, bonnet, trunk, door panels, fenders, bumpers), wheels, windows, lights and grille.

Regions with high textural courier $T_{R}>T_{S}$ (edge area) and bounded by low intensity courier $Y_{R}<Y_{S}$ (dark area) are defined as wheels, whereas regions with low intensity courier $Y_{R}<Y_{S}$ (dark area) and bounded by high textural courier $T_{R}>T_{S}$ (edge area) are defined as windows. Region with low textural courier $T_{R}<T_{S}$ (flat area) are defined as vehicle body. Finally, regions with high textural courier $T_{R}>T_{S}$ (edge area) are defined as lights and grille where light regions contain high intensity courier $Y_{R}>Y_{S}$ (bright area), while grille regions contain low intensity courier $Y_{R}<Y_{S}$ (dark area).

Once vehicle components are segmented, the geometric or transformed features of these components may be used to describe each component, while the topological relationships between components may also be used as additional feature dimension for identification.

\section{RESULTS AND DISCUSSIONS}

Over a hundred outdoor traffic image sequences on different roads have been captured under different

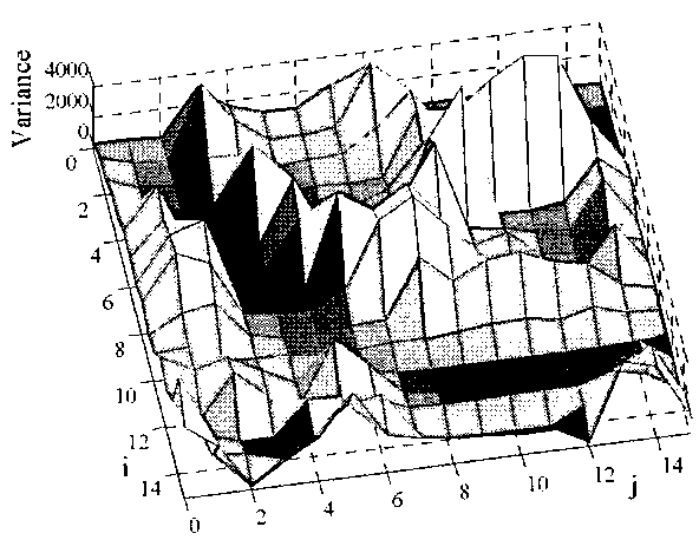

(a) Scale 4

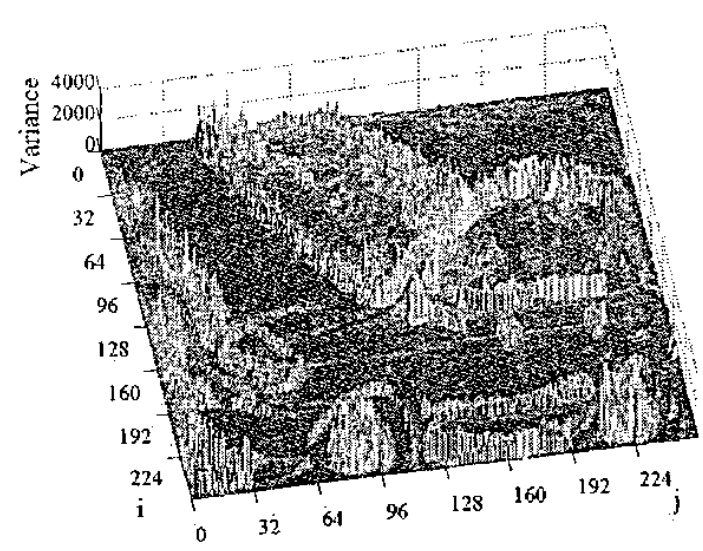

(b) Scale 8

Fig. 5: Texture couriers $T_{R}$

viewing angles, vehicle sizes and colors for testing the proposed method. Out of all tested images, a gray color sedan sample as depicted in Fig. 4(a) was chosen to illustrate the working of the proposed method. The corresponding background image, vehicle mask and scale 3 regions are depicted in Fig. 4(b), (c) and (d) respectively. With reference to Fig. 3 , the critical textural scale $C_{S}$ occurs at scale 6 . In order to show the trend of courier surfaces, Fig. 5(a)-(b) depict the surfaces of texture courier $T_{R}$ in 3D at scale 4 and scale 8 . As can be seen, scale 4 is too coarse to enable a good segmentation, while scale 8 contains too much detail, as discussed in Section 2.2. Fig. 6(a) and (b) depict the textural counier $T_{R}$ and intensity $Y_{R}$ courier at scale $6\left(C_{S}\right)$, respectively.

From Fig. 6, it can be derived, as illustrated in Fig. 7, those regions with high textural courier and bounded by low intensity courier are wheels. Regions with low intensity courier and bounded by high textural courier are windows. Regions of vehicle body enclose low textural courier. Regions of headlight contain high textural courier 


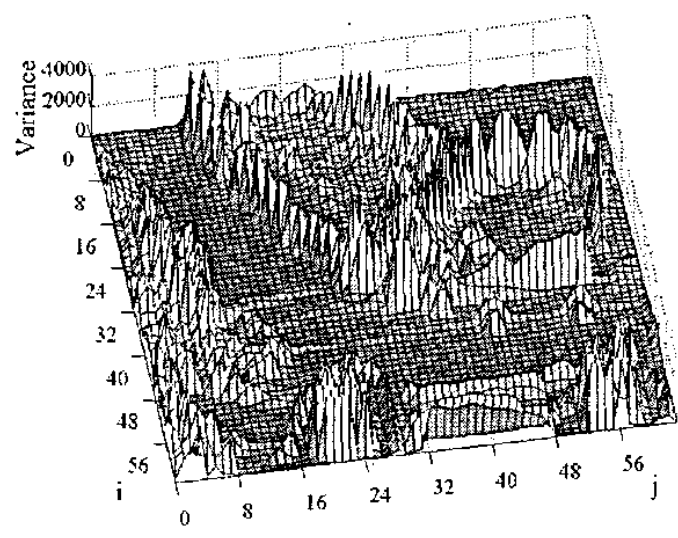

(a) Texture couriers $T_{R}$ at critical texture scale $C_{S}$

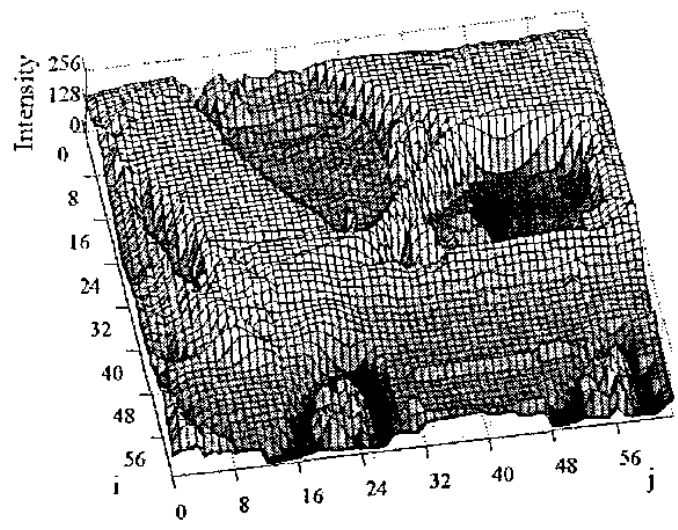

(b) Intensity couriers $Y_{R}$ at critical texture scale $C_{S}$

Fig. 6: Texture and intensity couriers

and high intensity courier while regions of grille contain high textural courier and low intensity courier. Detailed definitions of each vehicle component are summarized in Table 1. Having this vehicle components representation, further identification of vehicles can be made. For example the colors of two vehicle bodies can be easily compared based on low textural courier at the critical textural scale $C_{S}$.

\begin{tabular}{cc}
\hline Component & Region Definitions \\
\hline Wheels & $T_{R}>T_{S}$ bounded by $Y_{R}<Y_{S}$ \\
Windows & $Y_{R}<Y_{S}$ bounded by $T_{R}>T_{S}$ \\
Body & $T_{R}<T_{S}$ \\
Headlights & $T_{R}>T_{S}$ with $Y_{R}>Y_{S}$ \\
Grille & $T_{R}>T_{S}$ with $Y_{R}<Y_{S}$ \\
\hline
\end{tabular}

Table 1: Defined vehicle components based on $T_{R}$ and $Y_{R}$

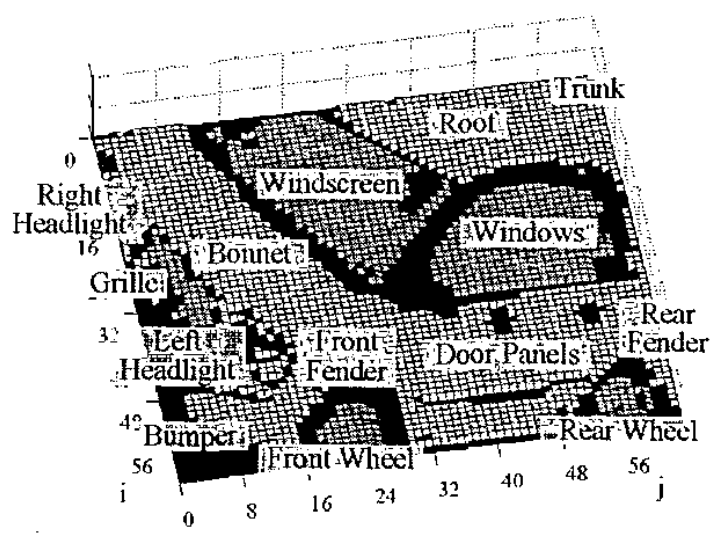

Fig. 7: Vehicle components as described at critical scale $C_{S}$

\section{CONCLUSIONS}

In this paper, we have presented a new scale space method for identifying vehicle components. In this method, textural difference in multi-scalc regions is the key to analyzing the vehicle image. Use in conjunction with the intensity difference, vehicle components can be differentiated. In further devclopment, velicle component details will be utilized for recognition of similar sized vehicles.

\section{REFERENCES}

[1] G.S.K. Fung, N.H.C. Yung and G.K.H. Pang, "Vehicle shape approximation from motion for visual traffic surveillance," IEEE Conference on Intelligent Transportation Systems, pp. 608-613, Aug. 2001.

[2] G.S.K. Fung, N.H.C. Yung, G.K.H. Pang and A.H.S. Lai, "Camera calibration from road land markings," Optical Engineering, vol. 42, no. 10, pp. 2967-2977, Oct. 2002.

[3] S. Gupte, O. Masoud and N.P. Papanikolopoulos, "Visionbased vehicle classification," IEEE Conference on Intelligent Transportation Systemis, pp. 46-51, Oct. 2000.

[4] A.H.S. Lai, G.S.K. Fung and N.H.C. Yung, "Vehicle type classification from visual-based dimension estimation," IEEE Conference on Intelligent Transportation Systems, pp. 203-208, Aug. 2001.

[5] W.W.L. Lam, C.C.C. Pang and N.H.C. Yung, "Highly accurate texture-based vehicle segmentation method," Optical Engineering, vol. 43, no. 3, pp. 591-603, Mar. 2004.

[6] T.N. Tan and K.D. Baker, "Efficient image gradient based vehicle localization," IEEE Transactions on Image Processing, vol. 9, no. 8, Aug. 2000.

[7] W. Wei, Q.S. Zhang and M. Wang, "A method of vehicle classification using models and neural networks," IEEE Conference on Vehicular Technologv, pp. 3022-3026, May 2001. 